\title{
DOI 10.18699/BGRS/SB-2020-202 \\ Identification of an AP2/ERF transcription factor controlling the synthesis of barley epicuticular wax
}

\author{
Ekaterina Kolosovskaya \\ ICG SB RAS, Novosibirsk, Russia \\ kolosovskaya@bionet.nsc.ru \\ Christian Hertig \\ Leibniz Institute of Plant Genetics and \\ Crop Plant Research (IPK) \\ Gatersleben, Germany \\ hertig@ipk-gatersleben.de \\ Dmitriy Domrachev \\ Novosibirsk Institute of Organic \\ Chemistry, SB RAS \\ Novosibirsk, Russia \\ dmitry@nioch.nsc.ru \\ Alexey Kochetov \\ ICG SB RAS, Novosibirsk, Russia \\ NSU, Novosibirsk, Russia \\ ak@bionet.nsc.ru
}

\author{
Sophia Gerasimova \\ ICG SB RAS, Novosibirsk, Russia \\ NSU, Novosibirsk, Russia \\ gerson@bionet.nsc.ru \\ Sergey Morozov \\ Novosibirsk Institute of Organic \\ Chemistry, SB RAS \\ Novosibirsk, Russia \\ moroz@nioch.nsc.ru \\ Vikhorev Alexander \\ ICG SB RAS, Novosibirsk, Russia \\ NSU, Novosibirsk, Russia \\ vikhorev@bionet.nsc.ru \\ Jochen Kumlehn \\ Leibniz Institute of Plant Genetics and \\ Crop Plant Research (IPK) \\ Gatersleben, Germany \\ kumlehn@ipk-gatersleben.de
}

\author{
Anna Korotkova \\ ICG SB RAS, Novosibirsk, Russia \\ korotkova@bionet.nsc.ru \\ Elena Chernyak \\ Novosibirsk Institute of Organic \\ Chemistry, SB RAS \\ Novosibirsk, Russia \\ chernyak@nioch.nsc.ru \\ Nikolay Shmakov \\ ICG SB RAS, Novosibirsk, Russia \\ shmakov@,bionet.nsc.ru \\ Elena Khlestkina \\ ICG SB RAS, Novosibirsk, Russia \\ NSU, Novosibirsk, Russia \\ Vavilov Institute of Plant Genetic \\ Resources (VIR) \\ Saint Petersburg, Russia \\ khlest@bionet.nsc.ru
}

\begin{abstract}
Site-directed mutagenesis provides ample opportunity for reverse genetics, which allows us to establish a relationship between a gene and its function. In the present study, the role of the HvWIN1 barley (Hordeum vulgare) gene in the formation of epicuticular wax was identified by taking a targeted knockout approach using RNA-guided Cas9 endonuclease technology.
\end{abstract}

Keywords - barley, Hordeum vulgare, genome editing, Cas9, gRNA, epicuticular wax

\section{Introduction}

Reverse genetics is a set of approaches aiming to establish a relationship between a gene and a trait. As its starting point, this strategy has a gene with an unknown function and finds out how a change in the structure or activity of the gene affects the phenotype. The use of novel methods facilitating site-specific mutagenesis opens up new prospects for solving the problems of reverse genetics. One of the directions in this area is the use of the Cas9/gRNA system to study the functions of regulatory genes. In the present work, the poorly studied HvWIN1 barley (Hordeum vulgare) gene, which encodes a transcription factor associated with resistance to biotic stress, was chosen as the target $[1]$. This gene belongs to the large family of plantspecific APETALA2/Ethylene-responsive factors (AP2/ERF) which play important roles in the regulation of many processes, including growth, development and stress response. In the present study, the targeted knockout of the HvWIN1 gene has led to phenotypic abnormalities. Normally, starting from the booting stage, the surface of the flag leaf sheath of barley is covered with a well-visible wax layer. Mutants exhibit a recessive phenotype of epicuticular wax deficiency. To reveal the role of the transcription factor HvWIN1 in the processes of epicuticular wax formation, a comparative microscopic, biochemical and transcriptome analysis of winl mutants vs wild- type plants was performed.
Materials and methods

Primary (M1=T0) barley mutants (cv. «Golden Promise») were obtained by targeted knockout of the HvWIN1 gene using the Cas9/gRNA system. Three TDNA-free mutant lines harboring different homozygous mutations in the HvWIN1 gene were selected in the M4 generation. The ultrastructure of the wax deposited at the plant's surface was studied using scanning electron microscopy. In addition, the biochemical composition of the epicuticular layer was evaluated using gas chromatography coupled with mass spectrometry. In order to identify genetic mechanisms of phenotype formation, a comparative transcriptome analysis of leaf blades and leaf sheaths of win1 mutant and wild-type plants was performed.

\section{Results}

Microphotographs and wax measurements showed that mutant plants and wild-type plants accumulate similar amounts of epicuticular wax on their leaf blades. By contrast, leaf sheath epicuticular wax of mutant plants differs remarkably from that of the wild-type in both total amount and composition. In particular, the amount of $\beta$ diketones is significantly reduced in mutants. An analysis of transcriptome data revealed that the mutation leads to a decrease in the activity of 326 genes and an increase of 292 in the leaf blade and a decrease of 63 and an increase of 967 in the leaf sheath. Among the genes showing reduced expression in the mutants were those previously known to be associated with the synthesis of epicuticular wax components. Among the genes that increased their expression in mutants, many are associated with cell wall biogenesis, modification and organization as well as with various responses to stresses, including pathogen attack and impact of reactive oxygen species. Additionally, many genes have been assigned to the gene ontology terms "extracellular space" and "cell membrane". 


\section{Conclusion}

The nature of the mutant phenotype and the data of comparative transcriptome analysis suggest that the transcription factor $H v W I N 1$ normally regulates the formation of the cuticular layer at the surface of leaf sheaths of upper barley leaves.

\section{Acknowledgment}

Supported by the RSF (16-14-00086). Research was conducted using equipment of The Core Facilities Center
"Microscopic analysis of biological objects" at the Central Siberian Botanical Garden SB RAS (Novosibirsk, Russia).

\section{References}

[1] A. Kumar, K.N. Yogendra, S. Karre, A.C. Kushalappa, Y. Dion, and T.M. Choo, "WAX INDUCERI (HvWINI) transcription factor regulates free fatty acid biosynthetic genes to reinforce cuticle to resist Fusarium head blight in barley spikelets", J Exp Bot., vol. 67(14), pp. 4127-39, July 2016. 